\title{
Clinical Trial Import-Export License Application
}

National Cancer Institute

\section{Source}

National Cancer Institute. Clinical Trial Import-Export License Application. NCI Thesaurus.

Code C115466.

An appeal made to one or more regulatory agencies requesting a license to import or export an investigational product, and/or clinical supplies. 